

\title{
A Statistical Analysis for the Car Key Fob Crowdsourced Design Evaluation Results based on the cDesign Framework
}

\author{
Hao $\mathrm{Wu}^{1}$, Jonathan Corney ${ }^{2}$ and Jing Gan $^{1}$ \\ ${ }^{1}$ Department of Industrial Design, School of Mechanical Engineering, Sichuan University, \\ Chengdu, China \\ ${ }^{2}$ Department of Design, Manufacture and Engineering Management, University of \\ Strathclyde, Glasgow, United Kingdom
}

\begin{abstract}
The "power of the crowd" has been repeatedly demonstrated and various Internet platforms have been used to applied collaborative intelligence to areas that range from open innovation to conceptual design. However, crowdsourcing applications in the fields of design research and creative innovation have been much slower to emerge. In this paper, the statistical analysis methods (i.e., normal probability plots) were used to validate the crowdsourced design evaluation results in the authors' previous crowdsourced design case study (i.e., car key fob design task). The discussion about the ranking distributions and results suggested that standard distribution trendlines and $\mathrm{r}^{2}$ results proved the effects of the ranking evaluation method used in the case study. The distribution determined that the evaluation process and results matched a normal distribution. As the contribution to knowledge, this paper applied the statistical analysis method to measure and approve the crowdsourced design evaluation method and its results.
\end{abstract}

Keywords: crowdsourcing, crowdsourced design (cDesign), crowdsourced design evaluation, statistical analysis, normal probability plots and trendlines,

\section{Introduction}

In the information age, design can be a product not only of individuals but may also result from the combined efforts of many people. Although such collaborative design systems are well documented in the literature for design activities carried out by, say teams of professional engineers and architects (Whitfield et al., 2002) less is known about the potential of distributed, anonymous, crowd-based collaboration in creative tasks. When crowdsourcing was defined and applied, this internet tool has become an effective tool in various research areas including, such as, linguistic study (Zaidan and Callison-burch, 2011), scientific research study (Buecheler et al., 2010), open innovation (Paulini, Murty and Maher, 2011) and of course collaborative design (Yu, Nickerson and Sakamoto, 2012)(Nickerson, Sakamoto and $\mathrm{Yu}$, no date). Based on the reported research of crowdsourced design, it can be found that the fundamental crowdsourced design framework has been built (Hao Wu, Corney and Grant, 2015). By using crowdsourcing as an effective tool all through design stages, and the methods for example, the Human-based Genetic Algorithms (HBGA) (Yu and Nickerson, 2011)(Hao; Wu, Corney and Grant, 2015) for creating designs, or Crowdsourced Design Evaluation Criteria 
(cDEC) for design evaluation process, the quality of final design outputs can be improved (the quality of design means the best designs selected from the crowd).

In this paper, the statistical analysis methods (i.e., Normal Probability Plot ${ }^{1}$ ) are used to investigate the statistical meaning of design measurement methods in the authors' reported case studies applied the Crowdsourced Design (cDesign) Framework (i.e., the car key fob design task (Wu and Corney, 2017)). Although the cDesign framework has been systematically applied in different origins of design tasks, the statistical meaning of this design evaluation process has not been determined. In this section, the authors give a brief introduction of crowdsourcing, crowdsourced design and the statistical analysis.

\subsection{Crowdsourcing}

In 2006, "crowdsourcing" was defined by Jeff Howe as "the act of a company or institution taking a function once performed by employees and outsourcing it to an undefined (and generally large) network of people in the form of an open call"(Howe, 2006). This new type of "crowd" is made up by anonymous groups (Yochai Benkler, 2006)(Howe, 2006). Crowdsourcing groups include online product communities (Brabham, 2009)(Jeppesen and Frederiksen, 2006)(Kozinets, Hemetsberger and Schau, 2008), virtual communities of special interests (Hogue, 2011), the general public (Chilton, 2009)(Haklay and Weber, 2008), and employees who typically would not participant in the tasks to be completed (Stewart, Huerta and Sader, 2009).

\subsection{Crowdsourced Design}

Then since 2006, the Human-based Genetic Algorithms (HBGA) has emerged as the principle way to support design using crowds (Yu and Nickerson, 2011)(Yu, Nickerson and Sakamoto, 2012)(Yu, 2011). HBGA requires designs to be combinable (i.e., merge distinct features) and also evaluable. In HBGA process, the heart is usually the combination of the best features from the 'parents'. In contrast to the established processes academic research into crowdsourced design has investigated the power of iteration, competition, reward and combination processes (Wu, Corney, \& Grant, 2014b; Lixiu Yu \& Nickerson, 2011), and the systematic framework (i.e., a design methodology) called cDesign (Crowdsourced Design) has been reported (Wu, Corney, \& Grant, 2015).

\subsection{Statistical Analysis}

In tradition, statistical analysis (the distribution of creativity in the population) has been discussed by Sternberg and Grigorenko (Sternberg and Grigorenko, 2003) as follows:

"Francis Galton (1986) first established that human abilities tend to be distributed in the population according to the 'normal' or 'bell-shaped' curve. His demonstration was based partly on data - the fit of the normal curve to performance on examinations - and partly on analogy to the distribution of physical traits, such as height and weight. Since Galton, the normal distribution has become almost an article of dogma, firmly ingrained in the statistics psychologists use and in their conception of individual differences, including intelligence (Burt, 1963). Moreover, it is clear that this faith is not unfounded, for the bell curve provides a reasonable approximation to most empirically observed distributions. Not surprisingly, creativity has often been perceived after the same fashion (Nicholls, 1972). Presumably, most human beings exhibit average levels of the capacity, the frequencies tapering off in either direction, with creative genius being about as rare as those who are virtually incapable of producing a creative idea."

\footnotetext{
${ }^{1}$ The normal probability plot is a graphical technique to identify substantive departures from normality. This includes identifying outliers, skewness, kurtosis, a need for transformations, and mixtures. Normal probability plots are made of raw data, residuals from model fits, and estimated parameters. More is shown in Wiki (1/2020):

https://en.wikipedia.org/wiki/Normal_probability_plot
} 
Then the exceptional individual who dominate some creative industries was discussed. However, since the crowd is drawn from the general population this argument for a skewed distribution are not relevant.

Although crowds can design (Yu and Nickerson, 2011)(Yu and Sakamoto, 2011)(Nickerson, Sakamoto and $\mathrm{Yu}, 2011$ ) and of course can evaluate designs (Bao, Sakamoto and Nickerson, 2011)(Herr et al., 2011), and even a cDEC method has been approved and reported that can be applied in the crowdsourced design process, the validation, or saying the measurement of this evaluation process itself has not been determined. Ultimately the results of a crowdsourced design methodology are critically dependent on the effectiveness of the evaluation process. Without an effective evaluation process the best designs cannot be reliably identified from the hundreds generated and consequently competition or HBGA approaches would perform poorly. The rest of this paper investigates the proper statistical analysis with the aim of assessing the effectiveness of an evaluation process.

So based on the above introductions, the hypothesis of the results is: the traditional statistical methods can be applied to validate the reported research for the evaluation process in the cDesign framework.

The following sections of this paper are as follows: in the next section (section 2), to understand the evaluation process of previous design experiments, it will be briefly described as two subsections: cDesign Framework and three case studies applying this framework. Then an introduction of the related existed statistical analysis methods is described in section 3, which is followed by the exact process of statistical analysing in the design task (i.e., car key fob design task), statistical analysis results and the discussion (section 4). Finally, this paper is ended with the conclusion section showing the summary of all findings in the paper, the limitation of this research and the suggested work in the future.

\section{Previous Research on cDesign}

\subsection{Crowdsourced Design (cDesign) Fundamental Framework}

The cDesign framework is briefly described in this section. In Figure 1, it is clearly illustrated that the cDesign Framework consists of four main stages: Specification, Prototype, Execution and Evaluation. The framework is used in this paper to establish the context of the authors' investigations (rather than being, say, a provable optimum model for crowdsourced design).

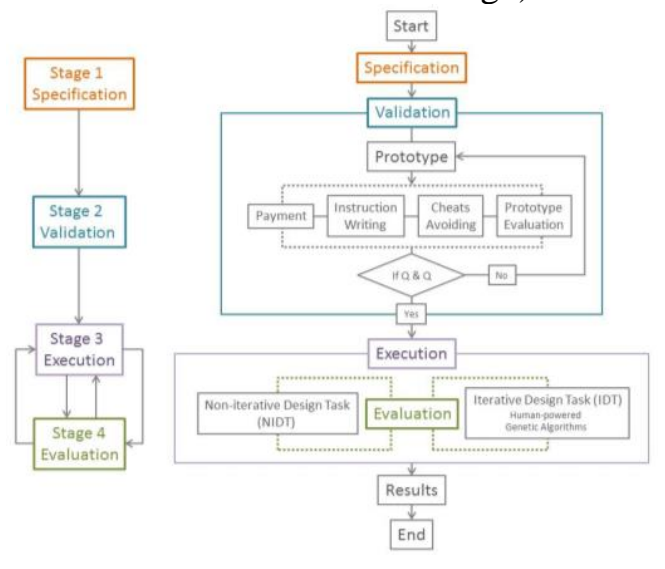

Figure 1. The cDesign Framework

\subsection{Design Case Study (Evaluation Process) - Car Key Fob Design Case Study}

The car key fob design experiment on mTurk has been reported (Wu and Corney, 2017). This design experiment applied the authors' cDesign Framework, and used the HBGA crowdsourced design method to systematically improve the design quality. Specially, the free-hand sketch design method is firstly in the cDesign framework based design process. Differently from other experiment used cDesign framework (Wu, Corney and Grant, 2014b)(Hao Wu, Corney and Grant, 2015)(Wu, Corney 
and Grant, 2014a), in this crowdsourced design task, because a free-hand sketch method was used to generate designs. The first step for participants of uploading their submission was to transfer their work into digital format (i.e., photo of sketch, scanned copy of sketch, etc.), then submitted them to participants.

In total, four generations of drawings were created by the crowd and evaluated. During the design creation stage, the best features from each pair of drawings were combined by the human workers to generate the new drawings. The process of evaluation and combination repeated to generate better quality of designs. The final evaluation shows that in this car key fob design task, the process resulted in improved conceptual design quality by a comparison between the last generation designs and the first generation designs. The results have not only demonstrated that the free-hand sketch method can be effectively used in a multi-stage (i.e., iterative) crowdsourcing process but have also provided a benchmark for the numbers of participants required to successfully carry-out a design task in this way. While many more trails will be required to establish if there is a general relationship exists between 'crowd size' and 'degree of design improvement', these results at least provide a first data point. Figure 2 shows some examples of the car key fob drawings in collected from design task.

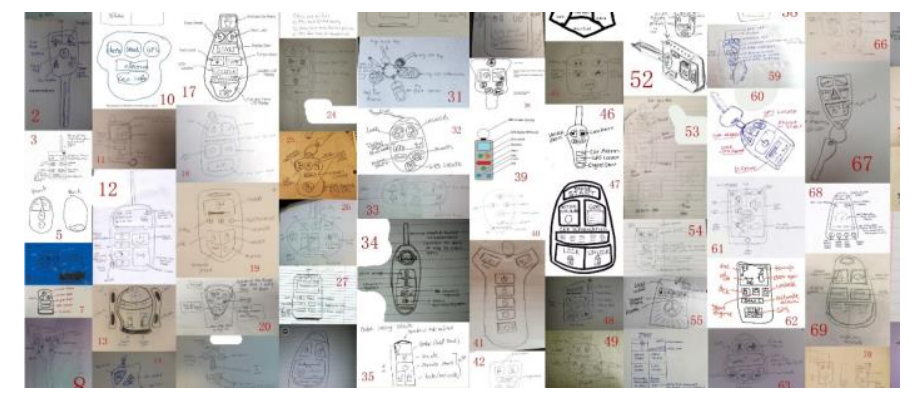

Figure 2. Examples of the drawings from the car key fob design task

\section{Statistical Analysis Methods}

To carry out the statistical analysis of the evaluation process and results from the case studies, the following statistical tools will be considered:

\section{Frequency Distribution}

There is academic evidence that design performance like many other human activities is normally distributed over the population (Runco, 2004). For example, figure 3 (left) illustrates a normal distribution, and figure 3 (middle) shows a random distribution in which the evaluation does not distinguish a standard performance.

\section{Trendline Chart/Normal Probability Plot}

The trendline chart is used to depict trends in the existing data or forecasts of future data. Figure 3 (fight) is an example of the trendline chart. In this chart, it can be observed that the trendline shows a normal distribution for the average ranking results, because $r^{2}=0.9699$ which is very close to 1 .


Figure 3. A normal distribution showing the design quality and the frequency (left), Evaluation does not distinguish a standard performance (middle), Trendline for the average ranking results (right) 


\section{Inverse Normal Distribution}

Inverse normal distribution establishes a normal distribution can be used in reverse to answer question such as:

If the quality of designs is normally distributed with an average of $\mu$ and a standard deviation of $\sigma$, calculate the number of designs required to produce a, say, $80 \%$ probability that the results include designs ranked in the top, say, $10 \%$.

\section{Discussions on the Statistical Analysis Results of the Case Study}

Figure 4 shows the process of the analysis.

In car key fob design task, when participants evaluated designs, it was required that all designs should be evaluated by marks: from 1 (the worst) to 100 (the best). However, because different people have different standards of judgements, the ranking of designs in individual groups was used to provide a relative ranking of design quality. In this section, the trendlines showing the distributions of these rankings will be described, and their significance discussed.

Table 1 shows the raw average data for all designs in the car key fob design tasks. Clearly illustrated in Figure 5 (left), (En-m in the figure means: the evaluation ranks for No.m design which is in the nth generation) in terms of the normal distribution plots, all ranking results from the 1st generation shows that the distribution is standard (in total, there were 17 groups of designs in the 1 st generation). The $\mathrm{r}^{2}$ information are shown in Table 2. It can be found that only two groups' $r^{2}$ are less than 0.9 (group 7 and 8 ). As for the 2 nd, 3rd and 4th generation evaluation ranking data, the normal distribution plots are shown in Figure 5 (right), and their $r^{2}$ are illustrated in Table 3. From the trendlines and $r^{2}$, it can be found that the distributions of the rank data are standard (only E2-2's $r^{2}=0.8736<0.9$ ).



Figure 4. Statistical Analysis General Process

\section{Conclusion, Limitation and Future work}

In this paper, the distribution of the evaluation ranking results was investigated. The discussions about the ranking distributions and results suggested that in the freehand sketch car key fob design task, standard distribution trendlines and $\mathrm{r}^{2}$ results proved the effects of the ranking evaluation. The distributions determined that the evaluation process and results matched a normal distribution.

However, as for the design experiment, it has been reported that the design case study has limitations (Wu and Corney, 2017). For example, although the experiments validate the effectiveness of cDesign Framework for concept sketches, it is still unknown that how a crowdsourced design process (structured by the cDesign Framework) will perform in a real product design projects. So in the future, to optimise the cDesign model, and to investigate new applications of crowdsourcing in design domain, for one thing, the cDesign framework should be applied in the real design projects (i.e., product design project) to validate its practicability; for another, new forms of crowdsourced design tools (i.e., mobile design tools) are required in this Internet and intelligence age. Finally, by collecting these new data and analyse the data statistically, is can be assumed that to receive the required quality of designs, the size of the crowd, the number of design samples or the time of collecting designs will be predictable. 
Table 1. Average ranking data for each generation in car key fob design task

\begin{tabular}{|c|c|c|c|c|c|c|c|c|c|c|}
\hline & E4-1 & E4-2 & E4-3 & E3-1 & E3-2 & E3-3 & E2-1 & E2-2 & E2-3 & E1-1 \\
\hline 1 & 1.625 & 1.5 & 2.625 & 3.5 & 1.875 & 1.5 & 2.625 & 2.5 & 1.75 & 2.375 \\
\hline 2 & 2.5 & 2.5 & 3.75 & 5 & 4 & 3.375 & 3.125 & 3.375 & 3.375 & 3.875 \\
\hline 3 & 4.25 & 4.375 & 4.5 & 5.25 & 4.625 & 3.875 & 3.5 & 4.25 & 3.875 & 4.5 \\
\hline 4 & 5.125 & 5.125 & 4.625 & 5.375 & 4.625 & 4.375 & 5 & 5.875 & 4.5 & 4.875 \\
\hline 5 & 5.25 & 6.25 & 4.625 & 5.625 & 5 & 5.375 & 5.125 & 6 & 4.875 & 5.625 \\
\hline 6 & 5.5 & 6.5 & 6 & 5.75 & 5.375 & 5.875 & 5.625 & 6.125 & 5.125 & 5.75 \\
\hline 7 & 5.75 & 6.625 & 6.25 & 5.875 & 5.375 & 6.625 & 6.25 & 6.25 & 6.625 & 6.125 \\
\hline 8 & 7.5 & 6.75 & 7.125 & 6.125 & 7 & 6.875 & 6.375 & 6.375 & 7 & 6.5 \\
\hline 9 & 8.5 & 7.625 & 7.5 & 6.5 & 8 & 8 & 7.75 & 6.5 & 8.375 & 6.875 \\
\hline 10 & 8.75 & 8.25 & 8.125 & 6.75 & 8.75 & 8.875 & 9 & 7.5 & 9.375 & 9.375 \\
\hline
\end{tabular}

\begin{tabular}{|c|c|c|c|c|c|c|c|c|c|c|}
\hline & E1-2 & E1-3 & E1-4 & E1-5 & E1-6 & E1-7 & E1-8 & E1-9 & E1-10 & E1-11 \\
\hline 1 & 3.375 & 3.125 & 2.625 & 2.25 & 2.5 & 2.75 & 1.625 & 1.125 & 2.25 & 2.5 \\
\hline 2 & 3.75 & 4.25 & 3 & 2.75 & 3.75 & 4.625 & 2.5 & 4.25 & 2.25 & 2.875 \\
\hline 3 & 4.5 & 4.5 & 4.25 & 2.875 & 4.25 & 4.75 & 4.75 & 4.375 & 3.5 & 3.75 \\
\hline 4 & 4.625 & 4.75 & 4.375 & 4.875 & 4.75 & 4.875 & 5.5 & 4.5 & 4.875 & 4.5 \\
\hline 5 & 4.75 & 5.5 & 4.75 & 5 & 5.5 & 5 & 6.125 & 4.75 & 5.75 & 5.125 \\
\hline 6 & 5.25 & 5.75 & 5.125 & 5.75 & 5.75 & 5.5 & 6.125 & 5.25 & 6.25 & 5.75 \\
\hline 7 & 5.5 & 5.875 & 6.375 & 6 & 6.125 & 5.5 & 6.25 & 7.25 & 6.375 & 7.375 \\
\hline 8 & 6.125 & 6.75 & 7.875 & 7.125 & 6.375 & 6.125 & 6.875 & 7.375 & 7.5 & 7.375 \\
\hline 9 & 7.75 & 7.75 & 8.125 & 8.875 & 7.5 & 6.25 & 7 & 7.5 & 7.625 & 7.625 \\
\hline 10 & 9.25 & 7.75 & 8.75 & 9.125 & 8.5 & 9.25 & 8 & 8.375 & 8.375 & 8.375 \\
\hline
\end{tabular}

\begin{tabular}{|c|c|c|c|c|c|c|c|}
\hline & E1-12 & E1-13 & E1-14 & E1-15 & E1-16 & E1-17 & Z-Score \\
\hline 1 & 2.25 & 3.375 & 2.875 & 2 & 3.375 & 2.375 & -1.64485 \\
\hline 2 & 3.25 & 3.375 & 3.25 & 2.625 & 4.375 & 2.875 & -1.03643 \\
\hline 3 & 4 & 3.625 & 3.75 & 3.625 & 4.375 & 3.875 & -0.67449 \\
\hline 4 & 4.75 & 4.125 & 4.75 & 4.625 & 4.625 & 4 & -0.38532 \\
\hline 5 & 5.125 & 5.375 & 4.875 & 4.625 & 5.25 & 5.625 & -0.12566 \\
\hline 6 & 5.5 & 5.625 & 5.625 & 6.125 & 5.625 & 6 & 0.125661 \\
\hline 7 & 6 & 6.125 & 5.625 & 7 & 6 & 6.625 & 0.38532 \\
\hline 8 & 6.375 & 7.375 & 6.75 & 7.375 & 6.375 & 6.75 & 0.67449 \\
\hline 9 & 7.75 & 8.125 & 7.75 & 8.375 & 7.625 & 6.875 & 1.036433 \\
\hline 10 & 9.75 & 8.375 & 9.25 & 9.375 & 7.875 & 9.625 & 1.644854 \\
\hline
\end{tabular}

Table 2. R2 information of the 1st generation evaluation ranks

\begin{tabular}{|c|c|c|c|c|c|c|c|c|c|}
\hline $\begin{array}{c}\text { Group } \\
\text { Number }\end{array}$ & E1-1 & E1-2 & E1-3 & E1-4 & E1-5 & E1-6 & E1-7 & E1-8 & E1-9 \\
\hline $\mathrm{R}^{2}$ & 0.9606 & 0.9031 & 0.966 & 0.9435 & 0.9481 & 0.9926 & 0.8578 & 0.8912 & 0.9108 \\
\hline $\begin{array}{l}\text { Group } \\
\text { Number }\end{array}$ & E1-10 & E1-11 & E1-12 & E1-13 & E1-14 & E1-15 & E1-16 & E1-17 & \\
\hline $\mathrm{R}^{2}$ & 0.9378 & 0.944 & 0.9740 & 0.9212 & 0.9609 & 0.9762 & 0.9624 & 0.9494 & \\
\hline
\end{tabular}


Table 3. R2 information of the 2nd, 3rd and 4th generation evaluation ranks

\begin{tabular}{|c|c|c|c|c|c|c|c|c|c|}
\hline $\begin{array}{c}\text { Group } \\
\text { Number }\end{array}$ & E2-1 & E2-2 & E2-3 & E3-1 & E3-2 & E3-3 & E4-1 & E4-2 & E4-3 \\
\hline $\mathrm{R}^{2}$ & 0.9716 & 0.8736 & 0.9813 & 0.9051 & 0.9484 & 0.9899 & 0.9578 & 0.9202 & 0.9687 \\
\hline
\end{tabular}

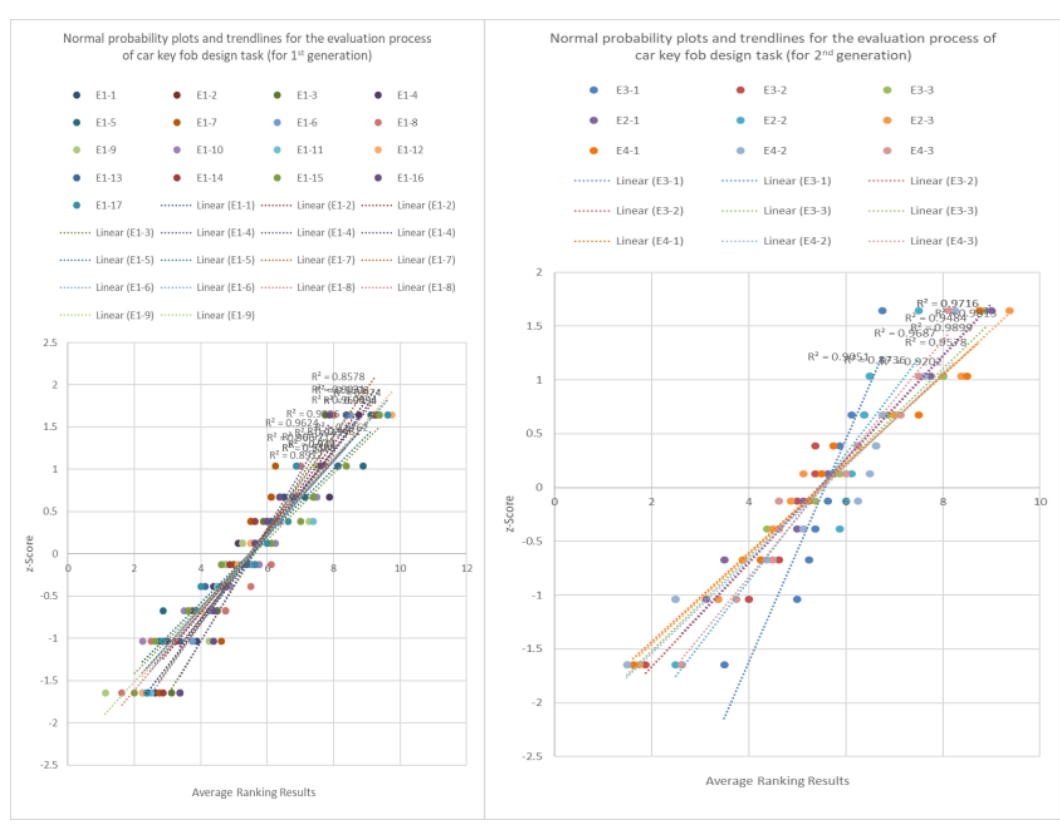

Figure 5. Figure caption The normal probability plots, trendlines and $\mathrm{r} 2$ information of the average ranking results in car key fob design task (1st generation) (left), The normal probability plots, trendlines and $\mathrm{r} 2$ information of the average ranking results in car key fob design task (2nd generation) (right)

\section{References}

Bao, J., Sakamoto, Y. and Nickerson, J. (2011) 'Evaluating Design Solutions Using Crowds', in Seventeenth Americas Conference on Information Systems, August 4th-7th, Detroit, Michigan. Available at: http://papers.ssrn.com/sol3/papers.cfm?abstract_id=2201651 (Accessed: 25 June 2013).

Brabham, D. (2009) 'Crowdsourced advertising: how we outperform Madison Avenue', Flow: A Critical Forum on Television and Media Culture. Available at:

http://scholar.google.com/scholar?hl=en\&btnG=Search\&q=intitle:Crowdsourced+Advertising:+How+We+Outp erform+Madison+Avenue\#0 (Accessed: 17 July 2013).

Buecheler, T. et al. (2010) 'Crowdsourcing, Open Innovation and Collective Intelligence in the Scientific Method: A Research Agenda and Operational Framework Why Crowdsourcing in the Scientific Method', in The Alife XII Conference. Odense, Denmark, pp. 679-686.

Chilton, S. (2009) 'Crowdsourcing is radically changing the geodata landscape: Case study of OpenStreetMap', 24th International Cartographic Conference. Available at: http://raptor1.bizlab.mtsu.edu/SDrive/DMORRELL/Mgmt 4990/Crowdsourcing/22_6.pdf (Accessed: 17 July 2013).

Haklay, M. and Weber, P. (2008) 'Openstreetmap: User-generated street maps', Pervasive Computing, IEEE, pp. 12-18. Available at: http://ieeexplore.ieee.org/xpls/abs_all.jsp?arnumber=4653466 (Accessed: 17 July 2013).

Herr, C. et al. (2011) 'THE ROLE OF COLLECTIVE INTELLIGENCE IN DESIGN', pp. 1-10. Available at: http://web.arch.usyd.edu.au/ mpau4467/papers/11_PauliniMurtyMaher.pdf (Accessed: 25 June 2013).

Hogue, C. (2011) 'Crowdsourcing for science', Chemical \& Engineering News. Available at: http://scholar.google.com/scholar?hl=en\&btnG=Search\&q=intitle:CROWDSOURCING+FOR+SCIENCE\#7 (Accessed: 17 July 2013).

Howe, J. (2006) 'The rise of crowdsourcing', Wired magazine, (14), pp. 1-5. Available at: http://sistemashumano-computacionais.wikidot.com/local--files/capitulo:redes-sociais/Howe_The_Rise_of_Crowdsourcing.pdf (Accessed: 22 June 2013). 
Jeppesen, L. B. and Frederiksen, L. (2006) 'Why Do Users Contribute to Firm-Hosted User Communities? The Case of Computer-Controlled Music Instruments', Organization Science, 17(1), pp. 45-63. doi:

10.1287 /orsc. 1050.0156 .

Kozinets, R. V., Hemetsberger, a. and Schau, H. J. (2008) 'The Wisdom of Consumer Crowds: Collective Innovation in the Age of Networked Marketing', Journal of Macromarketing, 28(4), pp. 339-354. doi: $10.1177 / 0276146708325382$.

Nickerson, J., Sakamoto, Y. and Yu, L. (2011) 'Structures for creativity: The crowdsourcing of design', ... Workshop on Crowdsourcing ..., pp. 1-4. Available at: http://crowdresearch.org/chi2011-

workshop/papers/nickerson.pdf (Accessed: 22 June 2013).

Nickerson, J., Sakamoto, Y. and Yu, L. (no date) 'Structures for creativity: The crowdsourcing of design', in CHI 2011 Workshop on Crowdsourcing and Human Computation: Systems, Studies, and Platforms, May 8, 2011, Vancouver, BC, Canada. Available at: http://crowdresearch.org/chi2011-workshop/papers/nickerson.pdf (Accessed: 25 June 2013).

Paulini, M., Murty, P. and Maher, M. (2011) 'Understanding collective design communication in open innovation communities', Journal of CoCreation in Design ..., pp. 1-13. Available at:

http://maryloumaher.net/Pubs/2011pdf/codesign_Draft_Oct2011.pdf (Accessed: 22 June 2013).

Runco, M. A. (2004) Everyone Has Creative Potential. Washington, DC: American Psychological Association. Sternberg, R. and Grigorenko, E. (2003) The Psychology of Abilities, Competencies, and Expertise. Cambridge University Press. Available at:

https://books.google.co.uk/books?id=9LEthsz9oCoC\&dq=normal+distribution+of+creativity+ability\&hl=zhCN\&source=gbs_navlinks_s.

Stewart, O., Huerta, J. and Sader, M. (2009) 'Designing crowdsourcing community for the enterprise', ACM SIGKDD Workshop on Human Computation. New York, New York, USA: ACM Press, pp. 50-53. doi: 10.1145/1600150.1600168.

Whitfield, R. et al. (2002) 'Distributed design coordination', Research in Engineering Design, 13, pp. $243-252$. doi: 10.1007/s00163-002-0021-1.

Wu, H. and Corney, J. (2017) 'A CROWDSOURCED DESIGN EXPERIMENT USING FREE- HAND SKETCH DESIGN METHOD BASED ON THE CDESIGN FRAMEWORK', in Proceedings of the 21st International Conference on Engineering Design (ICED 17), Vancouver, Canada, 21-25.08.2017, pp. 415-424. $\mathrm{Wu}$, H., Corney, J. and Grant, M. (2014a) 'Crowdsourcing Measures Of Design Quality', in 34th Computers and Information in Engineering Conference (CIE).

Wu, H., Corney, J. and Grant, M. (2014b) 'Relationship between quality and payment in crowdsourced design', in Proceedings of the 2014 IEEE 18th International Conference on Computer Supported Cooperative Work in Design (CSCWD). Ieee, pp. 499-504. doi: 10.1109/CSCWD.2014.6846895.

Wu, H., Corney, J. and Grant, M. (2015) ‘An evaluation methodology for crowdsourced design', Advanced Engineering Informatics. Elsevier Ltd, 29(4), pp. 775-786. doi: 10.1016/j.aei.2015.09.005.

Wu, H., Corney, J. and Grant, M. (2015) 'The Application of Crowdsourcing for 3D Interior Layout Design', Proceedings of the 20th International Conference on Engineering Design (ICED 15). Edited by C. Weber et al., Vol 4, pp. 123-134. Available at:

https://www.designsociety.org/publication/37769/the_application_of_crowdsourcing_for_3d_interior_layout_de sign.

Yochai Benkler (2006) 'The wealth of networks: How social production transforms markets and freedom', Yale University Press. Available at: http://paigrain.debatpublic.net/docs/1_Symposium_PFIE_6_2_web.pdf (Accessed: 17 July 2013).

Yu, L. (2011) 'Crowd creativity through combination', Proceedings of the 8th ACM conference on Creativity and cognition - C\&C '11. New York, New York, USA: ACM Press, p. 471. doi: 10.1145/2069618.2069756.

Yu, L. and Nickerson, J. (2011) 'Cooks or cobblers?: crowd creativity through combination', CHI 2011, May 712, Vancouver, BC, Canada. Available at: http://dl.acm.org/citation.cfm?id=1979147 (Accessed: 25 June 2013). Yu, L., Nickerson, J. and Sakamoto, Y. (2012) 'Collective Creativity: Where we are and where we might go', Proceedings of Collective .... Available at: http://papers.ssrn.com/sol3/papers.cfm?abstract_id=2037908 (Accessed: 25 June 2013).

Yu, L. and Sakamoto, Y. (2011) 'Feature selection in crowd creativity', Foundations of Augmented Cognition. Directing the ..., pp. 383-392. Available at: http://link.springer.com/chapter/10.1007/978-3-642-21852-1_45 (Accessed: 25 June 2013).

Zaidan, O. F. and Callison-burch, C. (2011) 'Crowdsourcing Translation: Professional Quality from NonProfessionals', in Proceedings of the 49th Annual Meeting of the Association for Computational Linguistics. 
Portland, pp. 1220-1229. Available at: http://delivery.acm.org/10.1145/2010000/2002626/p1220-

zaidan.pdf?ip=130.159.247.113\&id=2002626\&acc=OPEN\&key=C2D842D97AC95F7A.78A0E221B184F35C. 4D4702B0C3E38B35.6D218144511F3437\&CFID $=691357193 \&$ CFTOKEN $=37735314 \& \_$acm $\_=147853672$ 7_917ab1ef325b1c2acc6ff25e2e09. 